\title{
Osteoporosis Self Assessment Tool for Asian (OSTA) Index in Comparison to Quantitative Ultrasound of the Calcaneal in Predicting Low Bone Density.
}

\author{
Binod Sherchan', Arjun Lamichhane ${ }^{2}$, Deepak Prakash Mahara ${ }^{2}$. \\ ${ }^{1}$ Department of Orthopaedics, National Academy of Medical Sciences, Bir Hospital, \\ Kathmandu, ${ }^{2}$ Department of Orthopaedics, TU Teaching Hospital, Kathmandu.
}

\begin{abstract}
Introduction: Osteoporosis represent a worldwide public health problem, frequently resulting in fractures and leading to psychological problem, social consequences, functional limitation and poor quality of life. So it is important to identify those people who have high risk of osteoporosis, in order to reduce the incidence of osteoporotic fractures. The Osteoporosis Self-Assessment Tool for Asians (OSTA) index is a simple tool based on age and body weight. Calcaneal quantitative ultrasound (QUS) is another simple and low-cost instrument used to prescreen osteoporotic subjects. The aim of this study was to correlate between these two screening methods and to validate usefulness of Osteoporosis self assessment tool for Asian ( OSTA) in comparison with QUS of the calcaneum for the prediction of low bone density in Nepalese women.

Methods: This was a two-year descriptive observational study comprising 100 subjects performed at TU Teaching Hospital, Kathmandu, Nepal, from 2007 January to 2009 January who completed semi structured questionnaire with subsequent measurement of Quantitative Ultrasound (QUS) of the calcaneum. The sensitivity, specificity, and diagnostic accuracy of OSTA index was validated with the QUS T-score.
\end{abstract}

Results: When the risk category was defined as OSTA index $\leq-1$, and low QUS value as t-score $\leq-2.5$, the sensitivity, specificity and diagnostic accuracy of the index were $93.3 \%, 56.5 \%$, and $62 \%$ respectively, and the area under the curve was 0.7651 . When the low QUS value was taken as t score $\leq-1.0$, the sensitivity, specificity and diagnostic accuracy was $85.2 \%, 89.1 \%$ and $87 \%$ respectively.

Conclusions: The OSTA index, a simple and free risk assessment tool, can be used to estimate the prevalence of low QUS values in Nepalese women and may help to increase awareness and prevention of low bone mineral density.

Keywords: Bone Mineral Density; Osteoporosis; OSTA index; Quantitative ultrasound of calcaneum; T score.

\section{INTRODUCTION}

Osteoporosis represent a worldwide public health problem, affecting $45 \%$ of women who are fifty years of age or older resulting in life time risk of $40 \%$ for the fractures of hip, vertebrae, and distal forearm ${ }^{1}$. It is important to identify people who have high risk of osteoporosis, in order to reduce the incidence of osteoporotic fractures.

Dual Energy X-ray Absorptiometry (DEXA) is gold standard to Bone Mineral Density (BMD), however it is not readily available in Nepal and also is a costly method for screening purpose. Osteoporosis Self assessment Tool for Asian (OSTA) is a clinical decisions making risk index originally developed for the use in post menopausal Asian population ${ }^{2}$. It is an inexpensive, simple tool based on age and body weight. Purpose of OSTA index is not to diagnose osteoporosis or low BMD but to identify women who are more likely to have low BMD who could then undergo BMD

\section{Correspondence}

Dr. Binod Sherchan

Department of Orthopaedics, National Academy of Medical

Sciences, Bir Hospital, Kathmandu,

Email: drbsherchan@gmail.com 
measurement for a definitive assessment.

Calcaneal quantitative ultrasound (QUS) is another simple low cost, instrument use to screen osteoporotic subjects ${ }^{3}$.

The aim of this study was to correlate between these two screening methods and to validate usefulness of Osteoporosis self assessment tool for Asian ( OSTA) in comparison with QUS of the calcaneum for the prediction of low bone density in Nepalese women.

\section{METHODS}

It was a descriptive observational study conducted at Department of Orthopedics, TUTH from 2007 January to 2009 January. Any women age 40 or above with no previous diagnosis of osteoporosis with at least 1 additive risk for osteoporosis were included in this study. Previous diagnosis of osteoporosis and secondary osteoporosis were excluded from the study. Ethical clearance was taken from the ethical clearance committee. Informed consent from all the eligible patients was taken after full explanation about the nature of the study.

\section{Semi Structured Interview Schedule}

All participants completed a structured questionnaire. Information on demographic profile, age, age of menarche, age of menopause were obtained. Risk factors were stratified into non modifiable and modifiable. Non modifiable risk factors were defined by; age $\geq 65$ years, history of low energy fracture after the age of 40, history of Osteoporotic fracture in first degree relative and surgical menopause. Modifiable risk factors were defined by; smoking (current or past), weight of $127 \mathrm{lb}$ or less, early menopause [age $\leq 45$ yrs], prolonged premenopausal amenorrhea $[\geq 1 \mathrm{yr}]$ ), Late menarche (age 15 years or more), low calcium intake (lifelong), excessive alcohol intake ( $\geq 2$ drinks/ day), Excessive caffeine intake ( 2 to 4 cups/day) and inadequate physical activity. ${ }^{4,5}$

Sedentary lifestyle was defined as the occupation or activity that the subject engaged in most frequently in her life and accordingly subject was categorized as sedentary or heavy worker

\section{Anthropometry measurement}

Weight of the subject was measured in Kilogram with the standard measuring tool while subjects were standing, wearing light clothing and no shoes.

\section{Bone mass assessment}

Bone mass was assessed by broad band ultrasound attenuation using a QUS device. This device is small and portable, with a gel-coupled (dry) system that can measure BUA and speed of sound at the calcaneus. For all subjects, QUS was performed at the right calcaneus. The $T$-score for each subject was calculated by using the peak BUA value for a defined population of young adults ( ASIAN)

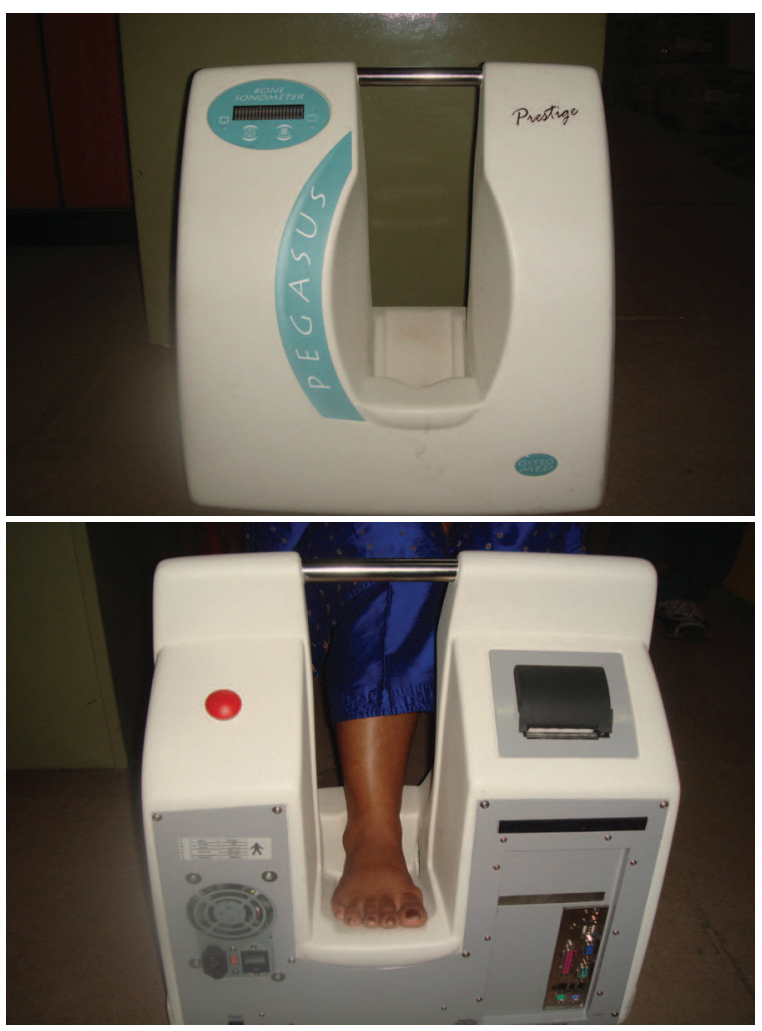

Figure 1. QUS device and Measurement of bone density

\section{OSTA INDEX CALCULATION}

OSTA Index $=($ Weight in kg- Age $) \times 0.2$

\section{OSTA RESEARCH GROUP CLASSIFI- CATION}

A. High risk subgroup (index $<-4$ )

B. Intermediate risk subgroup ( index -1 to -4 )

C. Low risk sub group ( index $>-1$ )

\section{Statistical analysis}

Here data are presented as percentage and as mean (standard deviation). Sensitivity, specificity, Diagnostic accuracy was calculated. Receiver operating curve analysis was performed and area under curve was calculated.To assess the internal validity of the index sensitivity was defined as the proportion of the subject with low $\mathrm{T}$ score correctly classified by the risk index 
(true positive) and specificity was defined as the proportion with normal $\mathrm{T}$ score correctly identified by the risk index (true negative) . ROC curve provided a graphical representation of the overall accuracy of a test by plotting sensitivity against (1- specificity) for all thresholds, while AUC quantified the accuracy of the test. All statistical analysis were performed by using SPSS software for Windows, version 11.5 (SPSS, Inc., Chicago, Illinois). with the mean index of -0.860 .

When the T- Score were calculated, 46 subjects were identified as Normal with mean T Score of 0.4089, 39 subjects were having Osteopenia with the mean $\mathrm{T}$ score of -1.7764 and 12 subjects were identified as Osteoporosis with the mean T- Score of -2.8792 . Only 3 were identified as severe osteoporosis with the mean T score of -3.1067 .

Table 1. Percentage of women identified with low bone density by QUS

\begin{tabular}{|l|l|l|l|}
$\begin{array}{l}\text { OSTA } \\
\text { Risk level }\end{array}$ & $\begin{array}{l}\text { Total number of } \\
\text { women }\end{array}$ & $\begin{array}{l}\text { No of women identified as } \\
\text { low QUS } \\
\text { T score } \leq-\mathbf{1 . 0}\end{array}$ & $\begin{array}{l}\text { of women out of each OSTA risk level } \\
\text { with low QUS } \\
\text { (T score <-1.0) }\end{array}$ \\
\hline High & 21 & 20 & $95 \%$ \\
\hline Medium & 30 & 26 & $86 \%$ \\
\hline Low & 49 & 8 & $16 \%$ \\
\hline
\end{tabular}

Table 2. OSTA index versus QUS when cut off $T$ score is taken as $\leq-2.5$

\begin{tabular}{|l|c|c|}
\multirow{2}{*}{ OSTA INDEX } & \multicolumn{2}{|c}{ QUS T score } \\
\cline { 2 - 3 } & With osteoporosis $($ T score $\leq-2.5)$ & With out osteoporosis $($ T score $>-2.5)$ \\
\cline { 2 - 3 } & $\mathrm{N}$ & $\mathrm{N}$ \\
\hline \multirow{2}{*}{ OSTA index $\leq-1$} & 14 & 37 \\
\hline OSTA index $>-1$ & 1 & 48 \\
\hline Total & 15 & 85 \\
\hline
\end{tabular}

Table 3. OSTA index versus QUS when cut off $T$ score is taken as $\leq-1.0$

\begin{tabular}{|l|c|c|}
\multirow{2}{*}{ OSTA INDEX } & \multicolumn{2}{|c|}{ QUS T score } \\
\cline { 2 - 3 } & Abnormal $(\mathrm{T}$ score $\leq-1.0)$ & Normal( T score $>-1.0)$ \\
\cline { 2 - 3 } & $\mathrm{N}$ & $\mathrm{N}$ \\
\hline OSTA index $\leq-1$ & 46 & 41 \\
\hline OSTA index $>-1$ & 8 & 46 \\
\hline Total & 54 & 5 \\
\hline
\end{tabular}

\section{RESULTS}

Mean age of the subject was 58.14 years with minimum age of 40 to maximum age of 84 years. Similarly mean weight of the subject was $53.91 \mathrm{~kg}$ with minimum weight of $28 \mathrm{~kg}$ to maximum weight of $92 \mathrm{~kg}$. Mean age of menopause was 46.60 years with the minimum age of 38 years to maximum age of 57 years.

The Osteoporosis Self Assessment Tool for the Asian was classified according to the original OSTA research group classification. A total of 21 subjects fell into OSTA class A with mean index of -5.590; 30 subject fell into OSTA class B with the mean index of -2.393 and 49 subject fell into OSTA class C (low risk subgroup)
When cut off point for the $\mathrm{T}$ score is taken as $\leq-1.0$, $95 \%$ of the subjects in High risk , $86 \%$ of the subjects in intermediate risk and $16 \%$ of the subjects in low risk group were identified as having low bone density (Table 1).

There were 14 true positive , 37 false positive, 1 false negative and 48 true negative cases. When the QUS T score cutoff value was taken as -2.5 the OSTA index has Sensitivity of $93.3 \%$, Specificity $56.5 \%$ and Diagnostic accuracy was $62 \%$ (Table 2 ). 
NOAJ July-December 2013|Vol 3| Issue 2

When QUS T score cutoff value was taken as $\leq-1.0$ , there were 46 true positive , 5 false positive , 8 false negative and 41 true negative cases and their Sensitivity was $85.2 \%$, Specificity was $89.1 \%$ and Diagnostic accuracy was $87 \%$ (Table 3 ). to 1999 among 4035 post menopausal women and they had High risk group comprising 11\%, intermediate risk group $47 \%$ and low risk group $42 \% .{ }^{6}$ Our study is comparable to their results in respect to the OSTA research group classification of the subject included in our study.

Table 4. variation in sensitivity, specificity and diagnostic accuracy at different $T$ score cutoff value

\begin{tabular}{|l|c|c|c|}
\hline T score cutoff & Sensitivity & Specificity & Diagnostic accuracy \\
\hline-2.5 & $93.3 \%$ & $56.5 \%$ & $62 \%$ \\
\hline-1.0 & $85.2 \%$ & $89.1 \%$ & $87 \%$ \\
\hline
\end{tabular}

T score cutoff value of -1.0 has better specificity and diagnostic accuracy than cutoff value of -2.5 (Table 4 ).

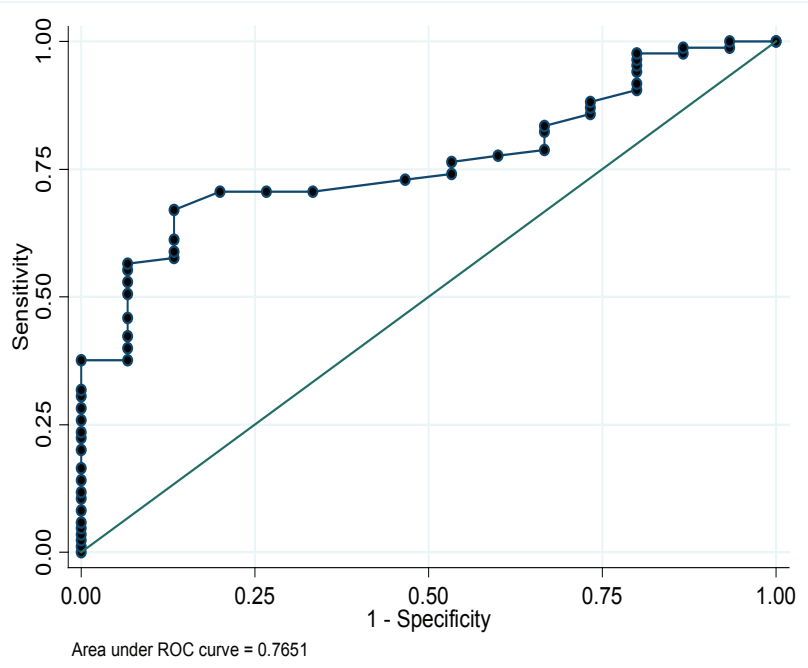

Figure 1. Receiver operating curve analysis

The sensitivity and specificity of the OSTA index in relation to $\mathrm{T}$ score obtained by QUS calcaneum were plotted as receiver-operating characteristic (ROC) curves. The areas under the curves (AUC) were calculated and the OSTA index provided AUC of 0.7651 (Figure 1). The results show that OSTA index is capable of selecting patients with low bone density as measured by QUS calcaneum.

\section{DISCUSSION}

Twenty one $(21 \%)$ of the subject fall into OSTA class A i.e. high risk sub group with mean index of -5.590 , a total of $30(30 \%)$ of the subject fall into OSTA class $\mathrm{B}$ i.e. intermediate risk group with the mean index of -2.393 and $49(49 \%)$ of the subject fall into OSTA class $\mathrm{C}$ i.e. low risk subgroup with the mean index of -0.860 .

F. Richy, et al performed a validation and comparative study of OST in Caucasian in Belgium between 1996
T- score quantifies the differences between the patients BMD and the mean value for young adults from the reference group. ${ }^{6,78}$ When the T- Score were calculated $46(46 \%)$ of the subject were identified as a Normal group with mean T Score of $0.4089,39(39 \%)$ of the subjects were identified as having Osteopenia with the mean T score of -1.7764 and $12(12 \%)$ of the subjects were identified as having Osteoporosis with the mean T- Score of -2.8792 . Only 3 (3\%)were identified as having severe osteoporosis with the mean $\mathrm{T}$ score of -3.1067. Anand et al from India in 2000 performed QUS calcaneum of 1713 subjects during a nine month period from sep 98 to may 99 . Using the WHO standard guideline they found that $48.9 \%$ of the subject were found to have bone mineral density within normal limit, $39.9 \%$ were found to have osteopenia and $11 \%$ were found to have osteoporosis. ${ }^{9} \mathrm{Vu}$ Thi Thu Hien, et al from Vietnam performed population based cross sectional survey at Hanoi city in 2003 and determined the crude prevalence of osteoporosis to be $15.4 \%$ when the QUS T-score cut off point was taken as $<-1.8 .{ }^{10}$ Our study has comparable results with other studies despite of relatively small sample size.

We validated the OSTA index with the two different cutoff $\mathrm{T}$ score value obtained by the QUS calcaneum. The abnormal value taken for OSTA index is $\leq-1$ as classified by the OSTA research group. When the cutoff $\mathrm{T}$ score value obtained by the QUS calcaneum is taken as $\leq-2.5$ as classified by WHO as a Osteoporosis, Sensitivity, Specificity and diagnostic accuracy of OSTA Index was $93.3 \%, 56.5 \%$, and $62 \%$ respectively. Similarly when the cutoff $\mathrm{T}$ score value obtained by the QUS calcaneum is taken as $\leq-1.0$ as classified by WHO as a osteopenia, Sensitivity , Specificity and diagnostic accuracy of OSTA Index was $85.2 \%, 89.1 \%$, and $87 \%$ respectively.

The sensitivity and specificity of the OSTA index in relation to T score obtained by QUS calcaneum were plotted as receiver-operating characteristic (ROC) curves. The areas under the curves (AUC) was 
calculated and the OSTA index provide AUC of 0.7651 and 0.8935 for $T$ score of -2.5 and -1.0 respectively. In a study conducted by Nan Ping Yong, et al in Taiwan in 2004 When the risk category was defined as OSTA index $\leq-1$, and low QUS value as $t$-score $\leq-2.5$, the sensitivity and the specificity of the index were $84.0 \%$ and $61.0 \%$, respectively, and the area under the curve was $0.81 .^{8}$

Hoon Choi, Yong Joo Park, Chul Min Lee, HongKyoon Lee from Korea showed that OSTA has $98.7 \%$ sensitivity and $73.4 \%$ specificity. ${ }^{11} \mathrm{~F}$ Richy et al in Belgium showed that OSTA has sensitivity of $97 \%$ to detect BMD $<-2.5$ with DEXA in a Hip. ${ }^{6}$

\section{H.M. Park, W. Ben Sedrine, J.-Y. Reginsterb, Philip} D. Ross from Korea validated OSTA index. In their study the OSTA had a high sensitivity (87\%), and good specificity $(67 \%)$ for identifying osteoporosis (DEXA BMD T-scores $\leq-2.5$ ). The prevalence of osteoporosis ranged from $2 \%$ among women classified as low risk $($ OSTA $>-1)$ to $64 \%$ among those classified as high risk $($ OSTA $<-4){ }^{12}$

Siris et al, in 2001 validated in Caucasian women using original SCORE population with 1102 post menopausal women aged 45 or more with sensitivity of $88 \%$ and specificity of $52 \%{ }^{1}$ In a population-based sample of postmenopausal Japanese women ${ }^{14}$, the OST had a sensitivity of $90 \%$ and specificity of $45 \%$. This tool was similarly validated in Philippine ${ }^{15}$

Studies have reported ranges varying from $24-95 \%$ and $56-93 \%$ respectively for sensitivity and specificity depending upon the various cutoff. ${ }^{16}$ Our result is similar to the other validational studies to exclude persons with low risk for osteoporosis. However there is a difference in the percentage of women identified as Osteoporosis in OSTA high risk group, which is less in our study than others. Similarly significant proportion of women in intermediate risk group had osteoporosis. This may be due to the fact that we had enrolled women age 40 years or above irrespective of menstrual status where as these studies mostly included post menopausal or older age group.

OSTA index value of $=$ or $<-1$ should be taken as a cutoff point to screen women so that maximum subjects with low bone density would be correctly identified and subjected to further evaluation. When cut off point for the $\mathrm{T}$ score is taken as $=$ or $<-1.0$, $95 \%$ of the subjects in High risk , $86 \%$ of the subjects in intermediate risk and $16 \%$ of the subjects in low risk group were identified as having low bone density.
Though the OSTA risk level classification had poor correlation with the classification with the WHO category based on T score, OSTA index cutoff value of -1.0 was equally good on identifying women with the low QUS value of -1.0.

\section{CONCLUSION}

OSTA index can be used as a first line screening tools in the clinic where detailed evaluation of osteoporosis is neither practical nor beneficial. OSTA cutoff value of $=$ or $<-1.0$ is the most accurate index in our study.

\section{REFERENCES}

1. Kevin B. Freedman et. al : treatment of osteoporosis: are physician missing an opportunity? Journal of Bone and Joint Surgery Am Volume 82-A, No 8 August 2000

2. Koh LK, Sedrine WB, Torralba TP, et al, Osteoporosis Self-Assessment Tool for Asians (OSTA) Research Group. A simple tool to identify Asian women at increased risk of osteoporosis. Osteoporos Int. 2001;12:699-705.

3. Claus-C, Gluer for the International quantitative ultrasound consensus group; Quantitative Ultrasound Techniques for the Assessment of Osteoporosis; Expert Agreement on Current Status, Journal of Bone and Mineral Research Vol, 12 Number 8, 1997

4. Cook RB, Collins D, Tucker J, Zioupos P;. Comparison of questionnaire and quantitative ultrasound techniques as screening tools for DXA.; Osteoporosis Int. 2005 May 10;

5. Hodgson SF, Watts NB, Bilezikian JP, et al. American Association of Clinical Endocrinologists: 2001 medical guidelines for clinical practice for the prevention and management of postmenopausal osteoporosis. Endocr Pract 2001; 7:293-312.

6. Richy F, Gourlay M, Ross PD, Sen SS, Radican L, De Ceulaer L, Ben Sedrine W, Ethgen O, Bruyere O, Reginster $J Y$ (2004) Validation and comparative evaluation of the osteoporosis self-assessment tool (OST) in a Caucasian population from Belgium. Q J Med 97:39-46

7. Larijani et al. Correlation of Quantitative Heel Ultrasonography With Central Dual-Energy X-ray Absorptiometric Bone Mineral Density in Postmenopausal Women J Ultrasound Med 2005;24:941-946.

8. Yang NP, Lin T, Wang CS, Chou P.; Correlation of osteoporosis screening by quantitative ultrasound of calcaneus and Osteoporosis Self-Assessment Tool for Asians in Taiwanese. J Formos Med Assoc. 2004 Feb;103(2):130-6

9. Anand L Parihar, Vandana M Dialini, Anant S Mashankar, Parag S Salkade, Manoj S Varma ; Quantitative ultrasound as a tool for assessment of bone status: an initial experience; Indian Journal of Radiology and Imaging 2000, 10:

10. Vu Thi Thu Hien, Nguyen Cong Khan, Nguyen Thi Lam, Le Bach Mai, DucSon NguyenTrung Le, Bui Thi Nhung, Masayo Nakamori Daisuke Kunii, Tohru Sakaiand Shigeru Yamamoto;Determining the Prevalence of Osteoporosis and 
Related Factors using Quantitative Ultrasound in Vietnamese Adult Women ; American Journal of Epidemiology 2005 161(9):824-830

11. Hoon Choi, Yong-Joo Park, Chul-Min Lee, Hong-Kyoon Lee; the validational and comparisional study of several risk indices for prediction of osteoporosis in peri-and postmenopausal Korean women, Dept of Obstetrics and Gynaecology, Inje University Sanggyepaik Hospital, Seoul, Korea

12. H.M. Park, W. Ben Sedrine, J.-Y. Reginsterb, Philip D. Ross ; Korean Experience With the OSTA Risk Index for Osteoporosis: A Validation Study; Journal of Clinical Densiometry; 2003 Vol 6 (3): 247-250

13. Siris ES, Miller PD, Barrett-Connor E, et al. Identification and fracture outcomes of undiagnosed low bone mineral density in postmenopausal women: results from the national osteoporosis risk assessment. JAMA 2001;286:2815-22.

14. Fujiwara S, Masunari N, Suzuki G et al. (2001) Performance of osteoporosis risk indices in a Japanese population. Curr Ther Res 62:586-594

15. Li-Yu JT, Llamado LJ, Torralba TP (2005) Validation of OSTA among Filipinos. Osteoporos Int Jul 19 [Epub ahead of print]

16. Eric J MacLaughlin et al; osteoporosis screening and education in community pharmacies using team approach ; Pharmacotherapy. 2005;25(3):379-386. (C2005 Pharmacotherapy Publications 